\title{
AVALIAÇÃO DE POPULAÇÕES DE PLANORBIDEOS, EM HABITATS NATURAIS, PELO MÉTODO DE PERFURAÇÃo DA CONCHA
}

\author{
Paulo Marcos Zech COELHo (1), Silvia Elizabeth GERKEN (1) \& José Rabelo de FREITAS (2)
}

\section{R E S U M O}

Propõe-se um novo método de marcação de planorbideos para determinação de densidade e de outros parâmetros pertinentes ao estudo de dinâmica de população, como capacidade de migração, taxas de crescimento e potencial biótico nos habitats naturais. Consiste em marcação através de dois furos de cerca de $2 \mathrm{~mm}$ de điāmetro de cada lado da concha em sua extremidade anterior, usandose um estilete metálico. Após a marcação os caramujos são imediatamente rein. troduzidos no habitat, em local correspondente ao da captura processada poucos minutos antes. Nos experimentos realizados, as taxas de caramujos marcados, recapturados uma semana após a marcação, variaram de 14 a $18 \%$ e permitiram calcular a população total de B. glabrata, de $5 \mathrm{~mm}$ de diâmetro ou mais. $O$ método permite estimar também a população de jovens com menos de $5 \mathrm{~mm}$ e fazer marcações múltiplas. É muito simples, de fácil manejo, não exige equipamentos nem pessoal especializados e provoca menos impacto no ambiente do que outros métodos.

UNITERMOS: Planorbideos - dinâmica de população - habitat natural - método de perfuração de concha.

\section{N T ROD U C A O}

O estudo đa dinâmica de populaçóes de planorbídeos requer técnicas acuradas para medidas de densidade e de outros parâmetros populacionais. Os métodos mais simples e os mais adotados em levantamentos epidemiológicos e de controle de populações de caramujos hospedeiros da esquistossomose, como o das conchadas e o de homens/hora de coleta, cumprem com bastante eficiência esses objetivos, mas carecem de precisão para as determinações de abundância. No entanto, têm sido muito usados para medidas de densidade, e taxas e frequiência e estrutura etária de populações malacologicas (STEPHENSON ${ }^{14}$; WATSON ${ }^{17}$; DOBROVOLNEY \& BARBOSA 5 ; PFLUGER ${ }^{13}$; POINTIER 12; WIBAUX.CHARLOIS et al. ${ }^{18}$; DENNIS ef al. ${ }^{4}$; GRISOLIA \& FREITAS ${ }^{6}$ ). OLI-
VIER e \& SCHNEIDERMAN 11 descrevem várias técnicas e instrumentos de coleta de caramujos, adotados desde a década de cinquenta até hoje. Métodos mais sensiveis para medidas quantitativas, baseados no método dos quadrados, têm sido usados nos últimos anos, princi. palmente em estudos de moluscos africanos (MAKIYA et al. ${ }^{10}$; TOMAS \& TAIT ${ }^{16}$ ). Tais métodos e outros mais sofisticados, como o dos marcadores radioativos, têm sido adotados em estudos ecológicos mais amplos (KNIGHT et al. 8; LEAL \& FRANCA ${ }^{7}$ ).

O objetivo deste trabalho i apresentar os resultados de um novo método baseado no processo de diluição por marcadores ecológicos. Visa determinar a densidade populacional, as ta.

(1) Departamento de Parasitologia, ICB - UFMG

(2) Departamento de Biologia Geral, ICB - UFMG 
COEL_FO, P. M. Z.; GERKEN, S. E. \& FREITAS, J. R. de - Avaliaçäo de populaçōes de planorbídeos, em habitats naturais, pelo método de perfuração da concha. Rev. Inst. Med. trop. São Paulo, 28:74-79, 1986.

xas de migração e de crescimento em habitat natural de caramujos hospedeiros da esquistossomose, de maneira mais simples e precisa, causando o menor impacto possível aos espécimens usados e ao habitat. O método pretende se apresentar como de fácil execução e dispen. sa treinamento especial de pessoal técnico.

\section{MATERIAL E METODOS}

\section{Area de estudo}

O projeto se desenvolveu na Lagoa do Diogo, pequena represa de forma arredondada, com largura máxima de $70 \mathrm{~m}$ e até 1,50 de profundidade. Situa-se junto ao $\mathrm{Km} 66$ da estrada Belo Horizonte - Itabira, logo após o trevo que segue para Monlevade. A margem direita da lagoa é coberta por uma faixa contínua de Telanthera sp que avança até 3 ou 4 metros da margem atingindo $0,50 \mathrm{~m}$ de profundidade. $\mathrm{Na}$ margem oposta a vegetação aquática é mais es. cassa, apresentando apenas alguns tufos esparsos de Telanthera sp. Na sua cabeceira ocorre uma comunidade compacta de Typha sp que cobre toda a parte superior da represa, onde a profundidade chega a até $0,70 \mathrm{~m}$. E abastecida por água que vem de uma nascente, passando por um galinheiro e pocilga, onde recebe uma grande carga orgânica de fezes de frangos, de patos, de porcos e restos de rações. Grande parte de detritos deste material fica retido na parte brejosa da entrada da represa.

Há um gradiente de matéria orgânica na represa. A maior concentração, acima de 40 $\mathrm{mg} / 1\left(\mathrm{KMnO}_{4}\right)$ foi detectada na cabeceira, junto à Typha sp, que retém os detritos. A matéria orgânica vai diminuindo na sua passagem pela comunidade de Telanthera sp, área de escoamento do fluxo principal das águas, onde ela cai de 40 para cerca de $20 \mathrm{mg} / 1$. Na margem oposta, a concentração de matéria orgânica foi 100 vezes menor que na área da Typha sp e o OD chegou a $100 \%$ de saturação, enquainto nas outras áreas atingiu apenas $30 \%$ de saturação. A alcalinidade na cabeceira foi em média de $44.0 \mathrm{mg} / 1$ de $\mathrm{CaCO}_{3}$, quase o dobro das outras áreas. $\mathrm{O}$ pH esteve próximo de 7,0 e a dureza em torno de $14 \mathrm{mg} / 1 \mathrm{em}$ toda a represa. Aliás, dureza e pH foram muito semelhantes nas três áreas. Nos levantamentos preliminares, B. glabrata esteve presente em todas as estações de coleta.

\section{Método de marcação dos caramujos}

Os caramujos foram coletados através de conchadas aleatórias em estações distantes entre si, cerca de $10 \mathrm{~m}$. Contavam-se todos os caramujos capturados, anotando-se os respectivos diâmetros de concha. Entretanto, só foram marcados aqueles com diâmetro superior a $5 \mathrm{~mm}$. A marcação foi feita através de perfuração de 2 furos em torno de $2 \mathrm{~mm}$ de diâmetro, um de cada lado, na parte anterior da concha, usando se, para tanto, um estilete. Os caramujos eram colocados sobre um pedaço de isopor, de madeira a ficarem com a parede da abertura da concha, a ser perfurada, firmemente apoiada no isopor, a fim de que a ação do estilete atingisse apenas a parte da concha a ser perfurada. Quando a parte mole do molusco se apresentava projetada para fora da concha, bastava um leve toque com o cabo do estilete para que esta se recolhesse, ficando assim a abertura desimpedida para o processamento dos furos. A operação é executada com luvas e pinças, para se evitar infecção acidental.

Após a marcação, os caramujos eram imediatamente devolvidos ao ambiente aquático, cuidando-se para que esta devolução correspondesse ao local de captura. Uma semana após, repetia-se a coleta por meio de concha. das, anotando-se os diâmetros de todos os caramujos capturados, como também o número daqueles marcados. Imediatamente, todos os individuos eram reintroduzidos nos locais on de a captura se processou.

A proporção de caramujos marcados em re. lação aos não marcados, permite, por regra de três simples, determinar, com boa precisão, o número total de caramujos do habitat considerado. A fórmula pode ser resumida assim:

Densidade populacional $=\frac{\mathrm{A} \times \mathrm{C}}{\mathrm{B}}$

(refere-se a caramujos com diâmetro da concha de $5 \mathrm{~mm}$ ou mais).

$A=$ Total de caramujos capturados, marcados e não marcados.

B $=$ Total de caramujos recapturados, marca dos.

$\mathrm{C}=$ Total de caramujos marcados introduzi dos no habitat logo após a marcação. 
COELHO, P. M. Z. GERKEN, S. E. \& FREITAS, J, R. de - Avaliação de populaçōes de planorbideos, em habitats naturais, pelo método de perfuração da concha. Rev. Inst. Med. trop. São Paulo, 28:74-79, 1986.

Como se conhecia a proporçăo dos caramujos com diâmetro de concha igual ou superior a $5 \mathrm{~mm}$, em relação à populaçăo total, pode-se perfeitamente determinar, com razoável precisão, a população total.

\section{RESULTADOS}

De 200 a 350 caramujos marcados e reintroduzidos no próprio habitat, foram recuperados, juntamente com caramujos não marcađos, 34 a 61 exemplares em cada um dos quatro experimentos realizados mensalmente. A taxa de recuperação de caramujos marcados foi de 14 a $18 \%$ em cada experimento. A população da área, considerando apenas os caramujos maiores que $5 \mathrm{~mm}$ de diâmetro, foi estimada em 2.456 exemplares em janeiro, 1.787 em fevereiro, caindo para 1.115 e 1.156 individuos nos meses de abril e maio, respectivamente (Tab. 1).

T A B E I A I

Avaliaçăo de densidade populacional de Biomphalaria glabrata em habitat natural, Lagoa do Diogo, MG

\begin{tabular}{|c|c|c|c|c|c|c|}
\hline \multirow{3}{*}{$\begin{array}{l}\text { Experimento } \\
\text { (N.A) }\end{array}$} & \multirow{3}{*}{ Mês } & \multirow{3}{*}{$\begin{array}{c}\text { Caramujos } \\
\text { marcados (*) } \\
\text { (N. }{ }^{\circ)}\end{array}$} & \multicolumn{3}{|c|}{ Caramujos capturados $\left({ }^{*}\right)$} & \multirow{3}{*}{$\begin{array}{c}\text { Populaçấa } \\
\text { estimsda } \\
(\mathrm{N}, 0)\end{array}$} \\
\hline & & & \multirow{2}{*}{$\begin{array}{l}\text { Marcados } \\
\text { e não mar- } \\
\text { cados (N.a) }\end{array}$} & \multirow{2}{*}{$\frac{\text { Marcados }}{\left(\mathrm{N} . \mathrm{O}^{\circ}\right)}$} & \multirow{2}{*}{$\frac{\text { Recapturados }}{(\%)}$} & \\
\hline & & & & & & \\
\hline 1 & jan. & 350 & 428 & 61 & 14 & 2.456 \\
\hline 2 & tev. & 250 & 243 & 34 & 14 & 1.787 \\
\hline 3 & abr. & 200 & 262 & 47 & 18 & 1.115 \\
\hline 4 & maio & 200 & 214 & 37 & 17 & 1.156 \\
\hline
\end{tabular}

(*) Diâmetro da concha com $5 \mathrm{~mm}$ ou mais

(**) Uma semana após a marcação e reposição no habitat

As. médias de diâmetros das conchas, em cada mês de coleta, variaram de 20.6 a $22.6 \mathrm{~mm}$. A distribuição dos caramujos de acordo com tanto os tamanhos dos caramujos marcados como o dos não marcados mantiveram as mesmas proporções, não só no periođo do experimento, como também nas diferentes estações de coleta.

\section{DISCUSSAO}

Muitos trabalhos de campo, envolvendo dados sobre densidade populacional, têm se contentado em realizar estimativas de densidade relativa, baseadas em amostras limitadas coletadas através de dragas, conchas, folhas de palmeiras, etc. Isto tem se dado tanto pela falta de técnicas mais acuradas para as medidas de densidade, como também pelas dificuldades advindo do comportamento dos moluscos. Estes, mesmo em habitats e reduzidas dimensões, tendem a ter distribuição não uniforme, devido a diferença de profundidade na água, distribuição de alimentação, quantidade de luz incidente, abundância da vegetação etc. Assim, peque nas amostras de uma população podem não ser indicadoras reais da densidade populacional e coletas sucessivas em uma pequena área de estudo podem ocasionar danos permanentes às comunidades aquáticas e por isso os dados podem sofrer influência do método.

Alguns métodos, como o dos quadrados, se usados adequadamente, podem dar informaçōes seguras sobre a população total. Mas, devido ao desconhecimento dos tipos de distribuiçăo espacial das populações, um grande número de quadrados tem de ser examinado. Amostragens devem ser tomadas em todas as comunidades aquáticas escolhidas, incluindo uma boa camada do solo. O método dos quadrados é muito laborioso, consome muito tempo e pode ocasionar a destruição de parcela ponderável da comunidade biótica. No entanto, vem sendo adotado para moluscos hospedeiros desde 1947 (STEPHENSON 14), Devido a essas desvantagens, foi praticamente abandonado até fins da década de setenta. Durante as últimas décadas, foram adotados métodos pouco sensivieis, mas bastante práticos para determinar a presença de moluscos, quer em levantamentos de fauna malacológica, quer em controle de densidade de população, antes e após o uso de molusco. cidas. Para atender a estes objetivos, o uso de drogas, conchas metálicas, homens/hora, folhas de palmeira, etc., se mostraram bastante eficientes (STEPHENSON 14; DOBROVOLNEY \& BARBOSA 5; OLIVER \& SCHNEIDERMAN II) No entanto, sua eficiência é duvidosa para de- 
COELHO. P. M. Z; GERKEN, S. E. \& FREITAS, J. R. de - Avaliaçāo de populaçōes de planorbideos, em habitats naturais, pelo método de perfuraçấo da concha. Rev. Inst. Med. trop. Sāo Paulo, 28:74.79, 1986.

terminar ou estimar densidade populacional, apesar de ainda estarem em uso em estudos epidemiológicos da esquistossomose. Sua eficiência qualitativa é inegável, mas a quantitativa deve ser encarada com reservas.

Os estudos de ecologia aquática atuais envolvem conhecimentos mais precisos da fauna bentônica. Procuram conhecer o papel de cada elemento na cadeia alimentar. Os moluscos, representantes de primeira ordem entre os consumidores, têm um papel muito importante no balanço trófico das comunidades aquáticas, inclusive nas suas relações simbióticas com as macrófitas. Em vista disso, passaram a ser estudados como um elemento trófico, cuja prođução secundária precisa ser melhor conhecida para se avaliar sua importância na comunidade biotica. Por isso, há hoje uma preocupação maior em determinar com mais precisão as densidades reais dos moluscos. Tanto assim, que voltaram a ser adotados novamente os métodos dos quadrados através do uso de cilindros, aros metálicos, corers, dragas de Ekmann etc. (BROWN 1; MAKIYA \& col. ${ }^{10}$; BROWN 2; THOMAS \& TAIT ${ }^{16)}$. O que evoluiu realmente foi o tratamento estatístico. Além disso, voltaram a ser usadas técnicas de coleta que permitam estabelecer o tipo de distribuição espacial da espécie estudada. Para isso, há uma contagem de moluscos de cada amostragem (concha, draga, cilindro, etc.). Este método, já adotado na década de quarenta (KUNTZ \& WELLS ${ }^{9}$ ), calculava a densidade populacional através do número médio de moluscos de cada conchada, é considerado moroso e não muito prático para estudos epidemiológicos. $\mathrm{O}$ método dos quadrados é bastante laborioso para simples determinação de flutuação de densidade ou de controle de população e não é tão expedito ou acurado para medidas de migração, de taxas de sobrevivência e de crescimento individual e de população, em habitats naturais, como os métodos de marcação e de recaptura. Dentre estes, os métodos de marcadores radioativos são os mais precisos e também os mais dispendiosos. Requerem instrumentos de custos elevados, pessoal e laboratorios especializados. Os radioisótopos entram na cadeia trófica e podem constituir, por isso, uma ameaça para os homens e toda a fauna; apesar das doses diminutas usadas e detectadas no inicio da cadeia alimentar. A marcação múltipla com tintas ou material equivalente (CARDOSO \& PFEIF.
FER ${ }^{3)}$ é de custo menor e de mais fácil aplicação do que as técnicas com radioisótopos, mas apresenta também suas limitações. Podem provocar exarcebação de predação dos indivíduos marcados, exigem maior manipulação dos moluscos e podem causar danos devido à uma possível toxidez das tintas usadas.

Săo, portanto, vários os problemas que envolvem a determinaçăo de densidade populacional de planorbídeos. Os diversos métodos descritos na literatura mostram sempre uma ou mais das seguintes limitaçōes: a) não podem ser usados indiscriminadamente em todos os habitats; b) nem sempre são adaptáveis às estimativas quantitativas; c) causam distúrbios no habitat; d) apresentam baixa sensibilidade de detecção de caramujos; e) os resultados não sāo reprođutíveis em coletas sucessivas; f) são dispendiosos e demandam muito trabalho $\mathrm{e}$ tempo para sua execução; g) necessitam de equipes bem treinadas; h) retiram espécimens do ambiente.

De acordo com a Organização Mundial de Saúde ${ }^{19}$, um método que se aproximaria do ideal para estudos epidemiológicos, seria aquele que reunisse as seguintes caracteristicas: a) revelasse com boa aproximação a população de caramujos; b) provocasse o menor distúrbio possível no habitat; c) não alterasse o número de caramujos por remoção de exemplares; d) requeresse equipamento barato e de fácil manejo; e) fosse de simples execução, não necessitando de equipes bem treinadas; f) não consumisse muito tempo em sua execução.

Acreditamos que o atual método desenvolvido neste trabalho se aproxima destas características estabelecidas.

O método, pelos resultados da Tabela I, mostra uma boa reprodutibilidade e constância na proporção de caramujos: $14 \%$ e $14 \%$ (janeiro e fevereiro) e $18 \%$ e $17 \%$ (abril e maio). Estes resultados refletem, nos chamados métodos de diluição, uma inferência matemática populacional bastante aproximada. Por outro lado, não causa grandes distúrbios no habitat, como também o número de caramujos no ambiente não é alterado, pois os caramujos coletados são imediatamente devolvidos ao ambiente. Tanto nas operações de marcação como nas de ava. liação o método exige equipamentos extremamente simples, é rápido na sua execuçăo e com uma ou duas demonstraçōes de campo 0 pes. 
COELFO, P. M. Z; GERKEN, S. E. \& FREITAS, J. R. de - Avaliaçăo de populaçöes de planorbideos, em habitats na turais, pelo método de perfuraçăo da concha. Rev. Inst. Med. trop. São Paulo, 28:74-79, 1986.

soal técnico torna-se perfeitamente conhecedor da metodologia.

O método pretende contribuir para os estudos de auto-ecologia dos planorbideos, pois sabe-se que vários problemas ainda persistem e necessitarão de respostas para um possível uso no controle da esquistossomose. Assim, não se sabe, por exemplo, o tipo de distribuição espacial mais comum de nossas espécies de caramujos, sua capacidade de dispersão, taxas de crescimento, etc. Técnicas mais precisas de avaliação de densidade, como as de dragas ou cilindros, associadas ao uso de caramujos marcados, permitirão um conhecimento melhor destes aspectos da ecologia. A marcação múltipla, com a combinação de furos de doís tipos em quatro áreas da concha, por exemplo, dará chances de se obter pelo menos oito (8) tipos de marcação com um só furo. Os experimentos conduzidos em aquários não demonstraram nenhuma alteração nas taxas de mortalidade como no comportamento biológico dos caramujos marcados quando comparados com os respectivos controles não marcados, mantidos em idênticas condições. A marcação se regenera dentro de um mês, deixanđo uma área transparente. Por isso e devido aos possíveis deslocamentos dos caramujos, as avaliaçōes devem ser feitas semanalmente.

\section{SUMMARY}

Evaluation of aquatic snail populations, under natural habitat conditions, by the method of perforation of the shell

A new method for labeling planorbidae to estimate the density and other parameters related with population studies, such as migration capacity, growth rates and biotic potential in natural habitats, is proposed. The snails are labelled with two small holes (2 mm diameter each) perforated in each side of the shell by means of a pointed metalic tool. After labeling, the snails are immediately put back in their habitat, at the same site where they were captured a few minutes ago. In all experiments carried out, the rates of labelled snails, recovered a week after labeling, varied from 14 to $18 \%$ and allowed to estimate the total population of B. glabrata, with $5 \mathrm{~mm}$ diameter or more. This method enables to evaluate juvenile population (with less than $5 \mathrm{~mm}$ ). as well as to perform different marks. It is very simple, ease of handling; it does not require either specialized personnel or equipment and, on the other hand, it causes less trouble in the habitat than other procedures do.

\section{REFERENCIAS BIBLIOGRAFICAS}

1. BROWN, K. M. - The daptative demography of four freshwater pulmonate snails. Evolution, 33: 417-432, 1979.

2. BROWN, K. M. - Resource overlap and competition in pond snails: an experimental analysis. Ecology. 63: $412-422,1982$.

3. CARDOSO, C. A. B. \& PFEIFFER, W. C. - Estimativas de parâmetros populacionais de caramujos vetores da esquistossomose. Cienc. e Cult., 31 (supl.): 423, 1979.

4. DENNIS, E.; VORKPOR, P.; HOLZER, B.; HANSON, A.; SALADIN, K. \& DEGREMONT, A. - Studies on the epidemiology of schistosomiasis in Liberia: the prevalence and intensity of schistosomal infections in Bong Country and the bionomics of the intermediate hosts. Acta trop. (Basel), 40: 205-229, 1983.

5. DOBROVOLNEY, C. X. \& BARBOSA, F. S. - Field trials of Sodium Pentachlorophenate as a molluscide in flowing waters in Brazil. Publ. Avuls. Xnst. Aggeu Magalhães, 2: 121-157, 1853.

6. GRISOLIA, M, L. \& FREITAS, J. R. - Caracteristi. cas físicas e químicas do habitat da Biomphalarja te. nagophila. Mem. Inst. Osw. Gruz, 1985 (em publica. 乌ั̆о).

7. LEAL, M. A. M. \& FRANCA, E, P. - Limites de distăncias de deteç̧ăo de caramujos Bilomphalaxia gla brata marcados' com $\mathrm{Sr}-85$, usando como absorvedores 0 ar, a água e axeia. Mern. Inst. Osw. Cruz, 79: 227. 231,1984

B. KNIGHT, W. B.; LIARD, F.; RITCHIE, L. S.; PEL LEGRINO, J. \& CHIRIBOGA, J. - Labeling of Blomphalaria glabrata and cercariae of Schistosoma mansoni with radioselenfum. Exp. Parasit., 22: 309-315, 1968.

9. KUNTZ, R. E. \& WELLS, W. H. - Laboratory and field evaluations of two dinitrophenols as molluscicides for control of schistosome vector in Egypt with em. phasis on importance of temperature. Am. Mid., 31: 784.824, 1851

10. MAKIYA, K.; TANAKA, H.; BANES, E. A.; BLAS. B.: KUMADA, N. \& SANTOS Jr., A. T. - Population studies on Oncomelania quadrasi, the snail intermediate host of Schistosoma japonicum, in the Philippines. J. exp. Med., 51: 179-185, 1981.

11. OLIVIER, L. \& SCHNEIDERMAN, M. - A method for estimating the density of aquatic snail population. Exp. Parasit., 42: 109-117, 1956.

12. POINTIER, J. P. - Etude de la croissance de Biom. phalaria glabrata, mollusque hôte intermediaire de a 
COELHO, P. M. Z.; GERKEN, S. E. \& FRETTAS, J. R. de - Avaliaçäo de populaçóes de planorbideos, em hsbitats naturals, pelo método de perfuraçio da concha. Rev. Inst. Med. trop. São Paulo, 28:74-79, 1986.

schistosomose Intestinale dans les forats Marecageuses a Pterocarpus de Guadaloupe (Antilles Françaises). Malacologia, 22: 395-398, 1982.

13. PFLUGER, W. - Ecological studies in Madagascar of Biomphaiaris pfeiftert, intermediate host of Schistoso. ms mansoni. I. Seasonal variations and epidemiological features in the endemic ares of Ambositra. Arch. Inst. Pasteur Madagascar, 45: 79-114, 1976

14. STEPHENSON, R. W. - Bilharziasis in the Gezira irrigated area of the Sudan. Trans, roy. Soc. trop. Med. Hys., 40: 479-494, 1947.

15. SMITH, V. G. F, - Distribution of snalls of medical and vetertnary importance in a organically polluted watercourse in Nigeria. Ann. trop. Med. Parasit., 36: $539-546$, 1982 .

16. THOMAS, J. D. \& TAIT, A. I. - Control of the snail hosts of schistosomiasis by environmental manipulation: a field and laboratory appraisal in the Ibadan area,
Nigeria. Phtl, Trans, roy. Sac. Lond, B, 305: 201-253, 1984.

17. WATSON, J. M. - Stuaies on bilharaiasis in Iraq. VI. Seasonal variations of the vector snail, Bultinus truncatus. J. Fac. Med. Baghdad, 15: 33-68, 1951.

18. WIBAUX.CHARLOIS, M.; YELNIK, A.; IBRAHIMA, H. ; SAMEEKOBO, A. \& RIPERT, Ch. - Etude épidémiolo. gique de la bilharziose a $\mathbf{S}$. haematobium dans le pé. rimètre rizicole de Yagoua (Nord - Camaroun) ii. Distribuition et écologie des hotes intermédiaires. Bull. Soc. Path. éxot., 75: 72-93, 1982.

19. WORLD HEALTH ORGANIZATION - Study group on the ecology of intermediate snail hosts of bilharziasis Wld. Hith. Org. techn. Rep. Ser., 120: 3.38, 1957.

Recebicio para publicação em 21/3/1985.

REVISTA DO INSTITUTO DE MEDICINA TROPICAL DE SAO PAULO

$$
\text { ASSINATURAS - } 1986 \text { - BRASIL }
$$

Para assinar a Revista do Instituto de Medicina Tropical de São Paulo envie cheque nominal cruzado no valor de Cz $\$ 100,00$ (cem cruzados), endereçado à:

Revista do Instituto de Medicina Tropical de Säo Paulo

Av. Dr. Enéas de Carvalho Aguiar, 470

05403 São Paulo - S.P. 